\title{
A Novel Parking Service Using Wireless Networks
}

\author{
Gongjun Yan, Michele C. Weigle, Stephan Olariu \\ Computer Science Department \\ Old Dominion University \\ Norfolk, VA 23529 \\ \{ygongjun, mweigle, olariu\}@cs.odu.edu
}

\begin{abstract}
Parking is costly and limited in almost every major city in the world. The misparking aggravates the competition of parking slots. Innovative parking systems for meeting near-term parking demand are needed. A probabilistic approach is addressed to model the misparking of the current parking system. To provide an intelligent parking services and to eliminate the misparking, a wireless-based parking service is proposed. The proposed system adopts the wireless network and sensor technologies to provide an intelligent and automatic parking service. The implementation and a probabilistic analysis of the new parking service are presented as well.
\end{abstract}

\section{INTRODUCTION}

Parking is limited in almost every major city in the world contributing to traffic congestion, air pollution, and driver frustration. For example, the Manhattan Central Business District (CBD) has 109,222 off-street public parking spots [1], for a ratio of approximately one off-street public spot for every $16 \mathrm{CBD}$ workers. Yet, often parking spots are wasted. In large parking lots, a driver may exit the lot without knowing about new spots that have just become vacant. Finding an empty parking spot may also lead to driver frustration if another car takes the spot before the driver can reach it.

Current parking system often allows drivers to freely park instead of assigning a specified parking slot to each vehicle. Therefore, parking site cannot collect accurate parking vacant slots. Drivers cannot reserve a specified slot. The orderless competition of parking slots results parking slot waste, long slot searching time, and frustrated user experience. A nature solution will number each slot and assign them to drivers. But a driver assigned with a parking slot $a$ can park at slot $b$ for his own convenience or purpose. Therefore, misparking will happen. Misparking can affect other driver's parking behavior.

Thus, innovative parking systems for meeting near-term parking demand are needed. With new technologies (for example, wireless communications, computer, control, and electronics technologies), intelligent service-oriented parking services can improve parking space utilization and improve driver experience. Our motivation therefore is to provide a novel and intelligent parking service. The contributions of this paper are 1) a probability model which analyzes the misparking in conventional parking service, 2) a wirelessbased parking service.

This work is supported in part by U.S.A NSF (CNS 0721586)

\section{RELATED WORK}

Cassady et al. address strategies for selecting a "good" parking space. [2]. A "good" parking space is defined in three aspects: the walking time of a driver, the driving time, and the time to reach the front door. A probabilistic approach is used to evaluate the three "good" performance metrics. This method used in this paper does not help to reduce slot searching time and the slot utilization on macrolevel. Beside, Cassady assumes the drivers' knowledge of space availability. This assumption is strong in conventional parking system. In our proposal, a probabilistic approach is used to evaluate the misparking behavior. Then we proposed a new parking system that can eliminates the misparking.

Caliskan et al. [3] proposes a parking system in which parking automats are the producers of resource reports. The infrastructure uses IEEE 802.11 to broadcast these reports as raw text packets. Received reports are integrated into a vehicle's cache. These reports are aggregated and disseminated among vehicles. The decision strategy of which parking lot is used is based on two influencing parameters: the age of a resource and the distance to a resource. But the detail of detect vehicles is not stated. In literature, several approaches are employed to detect reliable information. First wired sensors are widely used [4]: 1) inductive loops, 2) pneumatic road tubes, 3) magnetic sensors, 4) piezoelectric sensors, 5) weigh-in-motion systems. Wolff et al. [5] use the Earth's magnetic field to detect parking spaces. These devices or sensors are physically wired to the control computers. One shortcoming of the wired sensor systems is that long and complicated wiring is required from parking lots to the central control unit. Therefore some wireless sensors can be applied to the parking space detection. Tang et al. [6] developed such a system using Crossbow Mote products and the extended Crossbow XMesh network architecture. Benson et al. propose RF transceiver and antenna with an ATMega 128L micro-controller system [7]. Third, image processing is applied to detect the vehicles [8], [9]. Funck et al. uses images to detect the parking space [10]. However these methods may incorrectly detect parking vehicles. One example is that a vehicle temporally uses one slot to park in another slot, or that a vehicle just happens to be in the intersection of these sensors. Panayappan et al. [11] propose a parking system in VANET to locate the available parking lots and spots. This system uses roadside units to 
relay parking messages and GPS to locate vehicle position. Roadside units maintain the security certificates and parking information. The greedy drivers are prevented from gaining more advantage from the system by lying. But it is not always working. For example, a roadside attacker pretending to be a vehicle can reserve as many slots as he wishes. In our system, all the communications are triggered by physical pressure on belts and are enabled by short range signals.

\section{Numbered PARKing SERVice}

\section{A. First Solution}

Although Rényi's parking problem [12] state a random parking service, current parking lot is often slotted and each vehicle can park only on one slot. Figure 1 shows a structure of a parking site which includes several lots. Each slot is assigned to one vehicle. Drivers can park at any slots. But this strategy will cause several problems. First, parking spots are wasted. In large parking lots, a driver may exit the lot without knowing about new spots that have just become vacant. Second, finding an empty parking spot may also lead to driver frustration if another car takes the spot before the driver can reach it.

Therefore, reservation a numbered slot is our first solution. We assume each vehicle can park at an assigned slot which is reserved before the driver's arrival. Although rare, there usually are some drivers who will take a wrong slot (mispark) under certain circumstance. The misparking of one driver will cause other drivers' misparking behavior. We model the misparking through a probabilistic approach in the next section.

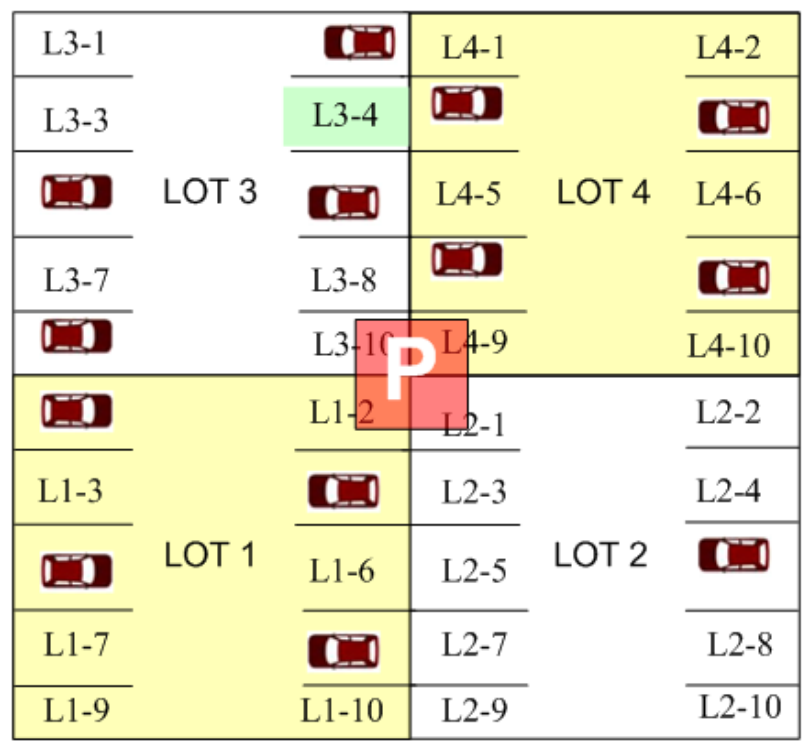

Fig. 1. Parking Spot Detail

\section{B. Probability Analysis}

Since misparking is often a small probability event, we present a probabilistic approach to analyze the effect caused by one misparking. We assume that each driver is assigned a parking slot. There is a parking lot of capacity $n$ where one of the customers misparks. Of interest is the probability that the last driver finds his assigned parking spot available. Let the $n,(n \geq 2)$, drivers be $P_{1}, P_{2}, \ldots, P_{n}$. Let $B_{k},(1 \geq k \geq n-1)$, be the event that $P_{k}$ is the first passenger who takes a wrong slot. We assume that $\operatorname{Pr}\left[B_{k}\right]=\frac{1}{n-1}$ independent of $k$. Let $A$ be the event that the last passenger finds her assigned slot available. By the Law of Total Probability

$$
\operatorname{Pr}[A]=\sum_{i=1}^{n-1} \operatorname{Pr}\left[A \mid B_{k}\right] \cdot \operatorname{Pr}\left[B_{k}\right]=\frac{1}{n-1} \sum_{i=1}^{n-1} \operatorname{Pr}\left[A \mid B_{k}\right]
$$

To find the probability of $\operatorname{Pr}[A]$, we will start a simpler problem which is stated in the lemma 1.

Lemma 1: $n,(n \geq 2)$, drivers park at a parking lot with capacity of $n$. Each driver is assigned a parking slot. The first driver takes the wrong slot. Subsequent drivers take their assigned slot if available, otherwise take an available slot at random. The probability that the last driver finds the assigned slot available is

$$
1-\frac{H_{n-1}}{n-1}
$$

Proof: Assume without loss of generality that the fist passenger $P_{1}$, with assigned slot $i$, takes slot $j$ instead.

For $2 \geq k \geq n$, let $S_{k}$ be the event that driver $P_{k}$ is assigned slot $j$. Let $A$ be the event that $P_{n}$ finds her assigned slot available. Clearly $\operatorname{Pr}[A]=\sum_{k=2}^{n} \operatorname{Pr}\left[A \mid S_{k}\right] \cdot \operatorname{Pr}\left[S_{k}\right]=$ $\frac{1}{n-1} \sum_{k=2}^{n} \operatorname{Pr}\left[A \mid S_{k}\right]$. For $2 \geq k \geq n$, write $q_{k}=\operatorname{Pr}\left[A \mid S_{k}\right]$; our goal now become to find a closed form for $q_{k}$.

Notice that $q_{n}=0$ and, more generally, for $2 \geq k \geq n-1$, $q_{k}=\frac{1}{n-k+1} \cdot 1+\frac{n-k}{n-k+1} \cdot q_{k+1}$. An easy inductive argument confirms that for $2 \geq k \geq n, q_{k}=\frac{n-k}{n-k+1}$, as shown in lemma 3.

It follows that

$$
\begin{aligned}
\operatorname{Pr}[A] & =\frac{1}{n-1} \sum_{k=2}^{n} \operatorname{Pr}\left[A \mid S_{k}\right] \\
& =\frac{1}{n-1} \sum_{k=2}^{n} \frac{n-k}{n-k+1} \\
& =\frac{1}{n-1} \sum_{k=2}^{n}\left(1-\frac{1}{n-k+1}\right) \\
& =1-\frac{1}{n-1} \sum_{k=2}^{n} \frac{1}{n-k+1} \\
& =1-\frac{H_{n-1}}{n-1}
\end{aligned}
$$

The probability that the last driver finds her assigned slot available is $1-\frac{H_{n-1}}{n-1}$.

Lemma 2: $q_{n}=0$ and, more generally, for $2 \geq k \geq n-1$, $q_{k}=\frac{1}{n-k+1} \cdot 1+\frac{n-k}{n-k+1} \cdot q_{k+1}$.

$$
q_{k}=\frac{n-k}{n-k+1}
$$

Proof: The proof is by induction on $k$. Basis step, $k=2$, $q_{2}=\frac{n-2}{n-1}$ because there are only $n-2$ possible correct slots can be taken by the last driver from the rest of $n-1$ untaken slots.

Inductive step, assume that $2 \geq k \geq n, q_{k}=\frac{n-k}{n-k+1}$. For 
$2 \geq k \geq n-1$

$$
\begin{aligned}
q_{k} & =\frac{1}{n-k+1} \cdot 1+\frac{n-k}{n-k+1} \cdot q_{k+1} \\
\frac{n-k}{n-k+1} & =\frac{1}{n-k+1} \cdot 1+\frac{n-k}{n-k+1} \cdot q_{k+1} \\
q_{k+1} & =\frac{n-k-1}{n-k}
\end{aligned}
$$

From the first principle of induction, we show that for $2 \geq$ $k \geq n, q_{k}=\frac{n-k}{n-k+1}$.

Lemma 3: $n,(n \geq 2)$, drivers park at a parking lot with capacity of $n$. Each driver is assigned a parking slot. The $k$ th driver takes the wrong slot. Subsequent drivers take their assigned slots if the slots are available, otherwise take an available slot at random. The probability that the last driver finds the assigned slot available is

$$
1-\frac{1}{2(n-1)}\left[H_{n-1}^{2}+\sum_{k=1}^{n-1} \frac{1}{k^{2}}\right]
$$

Proof: Recall that $B_{k}$ is the event that $P_{k}$ is the first driver who takes a wrong slot In fact, what we have computed in the previous lemma 1 was $\operatorname{Pr}\left[A \mid B_{1}\right]$ Now assume that $B_{k}, k>1$, holds; this implies that the previous drivers $P_{1}, P_{2}, \ldots, P_{k-1}$ took their assigned slots. We are faced with an instance of problem $\Pi(n-k)$. Reasoning as in the previous slide, we obtain $\operatorname{Pr}\left[A \mid B_{k}\right]=1-\frac{H_{n-k}}{n-k}$.

Thus,

$$
\begin{aligned}
\operatorname{Pr}[A] & =\frac{1}{n-1} \sum_{k=1}^{n-1} \operatorname{Pr}\left[A \mid B_{k}\right] \\
& =\frac{1}{n-1} \sum_{k=1}^{n-1}\left(1-\frac{H_{n-k}}{n-k}\right) \\
& =1-\frac{1}{n-1} \sum_{k=1}^{n-1} \frac{H_{n-k}}{n-k} \\
& =1-\frac{1}{n-1} \sum_{k=1}^{n-1} \frac{H_{k}}{k} \\
& =1-\frac{1}{2(n-1)}\left[H_{n-1}^{2}+\sum_{k=1}^{n-1} \frac{1}{k^{2}}\right]
\end{aligned}
$$

It is clear that $n \rightarrow \infty$ implies $\operatorname{Pr}[A] \rightarrow 1$. In term of our parking lot problem, as long as the parking lot is large, one misparked user has virtually no effect. We plot the two probability formulas in figure 2 . We notice that there is a gap between curves. The is because $\operatorname{Pr}[A] 2$ of the lemma 3 is an instance of problem $\Pi(n-k)$ which is $k$-slots less than the $\operatorname{Pr}[A] 1$ of lemma 1 . The number of parking capacity $n$ is the only parameter of $\operatorname{Pr}[A]$. If the parking capacity decreases, the probability $\operatorname{Pr}[A]$ decreases. If $n \rightarrow \infty, \operatorname{Pr}[A] \rightarrow 1$.

Lemma 4: $\operatorname{Pr}\left[A \mid B_{k}\right]=1-\frac{H_{n-k}}{n-k}$

Proof: Now assume that $B_{k}, k>1$, holds; this implies that the previous drivers $P_{1}, P_{2}, \ldots, P_{k-1}$ took their assigned slots. We are faced with an instance of problem $\Pi(n-k)$. We can map the $(n-k+1)$-th slot as the first slots in the problem $\Pi(n-k)$. Therefore, we can get this lemma by substituting $n$ in lemma 1 with $(n-k)$.

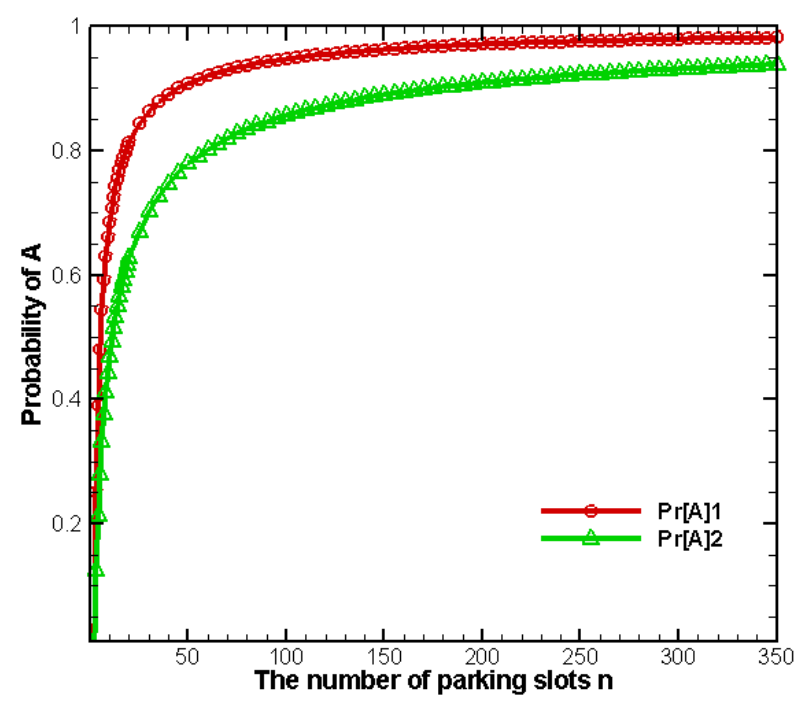

Fig. 2. The probability that the last driver finds the assigned slot available in a parking slot with $n$ slots. $\operatorname{Pr}[A] 1$ is from lemma $1, \operatorname{Pr}[A] 2$ is from lemma 3 ,

\section{WIRELESS-BASED PARKING SERVICE}

To provide an intelligent parking service and reduce or eliminate the misparking, we incorporate wireless and sensor technology to create a new parking service. The basic idea is the following. We assign each vehicle a parking card which is a parking permission to a specified slot. Sensors enlisted on the parking slot can detect the parking card to validate the misparking. If the vehicle is misparked, a report will be reported to maintenance people and the vehicle will be tolled away immediately by maintenance people. Sensors which form a belt on each parking slot are connected with a wireless transceiver which can communicate with the access point of parking service server. Therefore the server can figure out the empty slots and empty capacity of the parking site. The parking capacity information can be published to vehicles nearby through wireless networks. Vehicles can book a parking slot before they arrive at the parking lot. When a vehicle arrives and checks in at the parking booth, the vehicle will be assigned a parking card. The card records the transaction information, parking slot reservation, and a unique id.

\section{A. Infrastructure and Vehicular Model}

The infrastructure includes a parking service server, $n$ parking slots, $n$ infrared scanners and notice lights, $n$ sensor belts. As shown in figure 3, each slot embeds a sensor belt and an infrared pole. Pressure sensors are embedded inside the sensor belt. An infrared pole has a scanner which can scan the parking card inside the vehicle. If the scanner did not detect the parking card, a alarming light/voice can remind user to place the parking card at proper place inside the vehicle. 


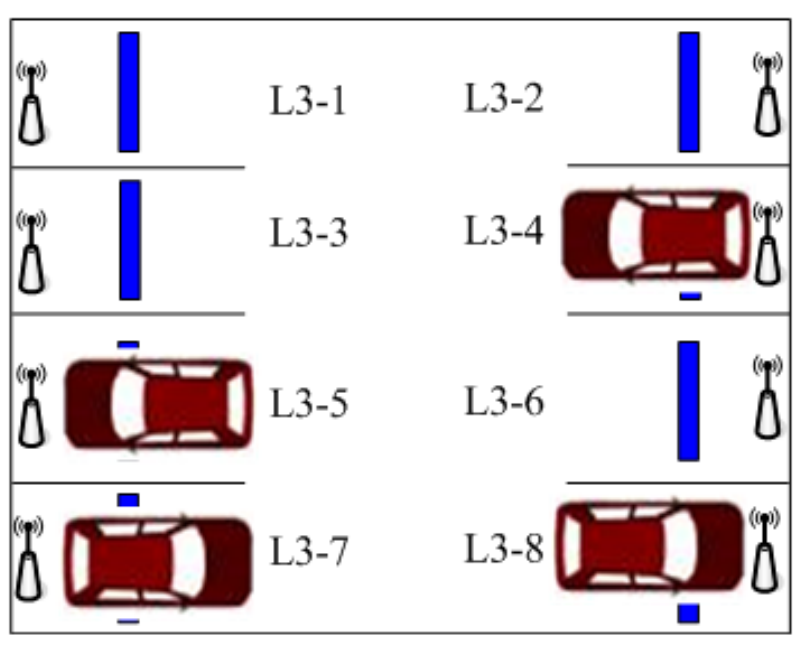

Fig. 3. Parking Spot Detail

\section{B. Wireless Communications}

1) Slot to Vehicle: The communication between slot and vehicles is shown in figure 4 . When a vehicle's wheels press on the sensor belt, the sensor belt will be activated and send signal to the infrared pole. The pole will turn on the infrared scanner to detect the parking card. After the authentication, the infrared pole will read the parking information: a temporal card ID, the reserved slot, a reservation timestamp, and transaction information. The temporal card ID is random unique number of the card. The reserved slot and reservation timestamp are used for the reservation of parking slot because the reservations from different places and users may collide. The transaction information indicates the fee, discount, payment, etc.

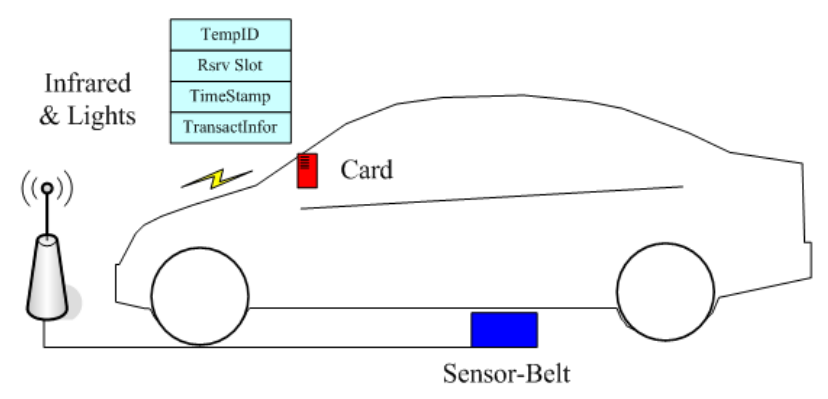

Fig. 4. Parking Spot Detail

If the scanner does not read the parking card, an alarming light and voice will turn on to indicate user to place the parking card in correct place where the scanner can read. If the parking card is read but parking information is wrong, the alarming light and voice will inform user the misparking. If the user ignores the alarming information, a message will be report to maintenance people and the vehicle of the user will be tolled away by maintenance people. In this way, we prevent misparking in our system.
2) Slot to Server: Drivers usually want to reserve a parking slot before they arrive at the parking lot. They even can book a parking slot while they are heading to the parking lot. To provide parking reservation service, we need to collect the empty slots. By using wireless network, each infrared pole can report to server that its slot is taken or not. The server will update its parking table and figure out the empty parking slots.

3) Server to Booth: When vehicles check in, parking lot booth will assign vehicles a parking slot. If a vehicle reserves slot before arriving, the vehicle will be assigned with the reserved slot. If a vehicle does not reserve slot before arriving, the vehicle will be assigned with a random empty slot. Therefore, the booth needs the synchronized parking capacity from the server. The communication between the server and the booth is base on wireless network.

4) Server to Vehicle: To inform vehicles near the parking lot, the server will broadcast parking capacity through wireless network or wireless radio. Vehicles can reserve parking slot through their terminal devices. The terminal devices can be personal computer, laptop, PDA, cell phone, telephones, etc.

\section{Parking Maintenance}

The parking maintenance work includes the routine check on electronics, clearance of misparking vehicles, etc. To ensure the function of the system, we have to maintain the system. If the parking lot is at a busy place, vehicles are coming and going all the time, we do not want to close the system to do maintenance work on a fixed time table. We can model the parking service and find a proper time to maintain the system.

Suppose the vehicles' arrival rate to the parking site is $\lambda$. Before maintaining the parking lot, we wait until we find no vehicles will come in next $T$ time units. Of interest is the expected time we have to wait before the maintenance work can start.

Assume we start to count vehicles at time 0 and let $X_{1}, X_{2}, \ldots$ the vehicle inter-arrival times. Let, further, $W$ be the random variable that counts the vehicles that will come before we can start the maintenance work. We model this problem as geometric distribution of Bernoulli trials: find the first $T$ which is larger than vehicle inter-arrival time. We write

$$
\begin{aligned}
\operatorname{Pr}[\{W=k\}] & =\operatorname{Pr}\left[\left\{X_{1}<T\right\} \cap\left\{X_{2}<T\right\} \cap \ldots \cap\left\{X_{k}<T\right\}\right. \\
& \left.\cap\left\{X_{k+1} \geq T\right\}\right] \\
& =\operatorname{Pr}\left[\left\{X_{1}<T\right\}\right] \cdot \operatorname{Pr}\left[\left\{X_{2}<T\right\}\right] \cdot \ldots \cdot \operatorname{Pr}\left[\left\{X_{k}<T\right\}\right] \\
& \cdot \operatorname{Pr}\left[\left\{X_{k+1} \geq T\right\}\right] \\
& =\left(1-e^{-\lambda T}\right)^{k} e^{-\lambda T}
\end{aligned}
$$

Thus, the expected number of vehicles that come before we 
can start maintenance is

$$
\begin{aligned}
E[W] & =\sum_{k \geq 0} k\left(1-e^{-\lambda T}\right)^{k} e^{-\lambda T} \\
& =\left(1-e^{-\lambda T}\right) e^{-\lambda T} \sum_{k \geq 0} k\left(1-e^{-\lambda T}\right)^{k-1} \\
& =\left(1-e^{-\lambda T}\right) e^{-\lambda T} e^{2 \lambda T} \\
& =e^{\lambda T}-1
\end{aligned}
$$

Finally, the expected time that we have to wait until we can start maintenance work is

$$
E[W] E[X]=\frac{e^{\lambda T}-1}{\lambda}
$$

Therefore, the expected time we have to wait before the maintenance work can start is (2).

\section{SIMULATION RESULTS}

In the simulation, we compare two scenarios. In scenario one, drivers randomly select a parking spot. The parking spot is based on first-come-first-serve. The process that drivers arrive at or exit to the parking lot is a Poisson Process. If they move around the parking area and can not find a parking spot, they turn around. If they turn around 3 times, they exit. In scenario two, drivers reserve a parking spot. The process that drivers arrive at or exit to the parking lot is the same Poisson Process. We assume 1\% of drivers will disobey the reservation and take somebody else's parking spot for their own convenience. The remaining $99 \%$ of drivers obey the reservation. We compare the parking utilization and average waiting time for drivers. The parking capacity is 1000 parking spots. The average arrival rate is 0.5 vehicle/min [13]. The simulation parameters and values are listed in Table I.

TABLE I

PARAMETERS AND VALUES

\begin{tabular}{|c|c|}
\hline Parameters & Values \\
\hline Exit booth service rate & 5 vehicles/min \\
Entry booth service rate & 20 vehicles/min \\
Number of exit booths & 5 \\
Number of entry booths & 1 \\
Average speed & $10 \mathrm{~km} / \mathrm{h}$ \\
Average arrival rate & 0.5 vehicle $/ \mathrm{min}$ \\
Average exit rate & 0.5 vehicle $/ \mathrm{min}$ \\
Average parking time & $1.5 \mathrm{hour}$ \\
\hline
\end{tabular}

We compared the conventional parking system with the proposed parking system. We varied the number of slots from 100 to 1000 in three comparisons: slot searching time, lot utilization, and average number of the misparked vehicles. Of interest first is the parking searching time because it is frustrated that we spend a lot of time in searching a parking slot in real life. We start to count the time spending in searching slot until a slot is found. As shown in figure 5(a), the proposed system spends much less time than the conventional system this is because the proposed system assigns each vehicle a slot when vehicle checks in. Vehicles directly head to the slot. We also investigated the parking utilization. After parking system become stable (the number of vacant slots stays in a stable status), we count the number of vacant slots. We get the slot utilization by calculating the percentage of vacant slots. As shown in figure 5(b), the conventional system has lower slot utilization than the proposed system. There are some vacant slots but vehicles do not find. In the proposed system, the service server knows the vacant slots which can be assigned to next coming vehicles. Moreover, we compared the average misparked vehicles. We collected the mispark vehicles every 30 minutes. The comparison result is shown in figure 5(c). As expected, the proposed system almost reduces the number of misparked vehicles to zero. But the conventional system obtains more misparked vehicles while the number of slots increases.

We examined our analysis of maintenance time as well. Three metrics are examined. They are the expected waiting time, the expected waiting number of vehicles, and the probability of finding the first maintenance time. We varied the maintenance time $T$ from 1 to 3 hours. We collected the average waiting number of vehicles, the average waiting time, and the $k$-th vehicle after which we found the maintenance time. The result is shown in figure 6(a), 6(b), and $6(c)$. The relationship among the three figures matches with the probability analysis shown in section IV-C.

\section{CONCLUSIONS AND FUTURE WORK}

This paper has proposed a wireless-base parking system based on the probability analysis of the conventional parking system. By using the wireless network and sensors, the proposed parking system can provide intelligent parking services to drivers. Simulations are comparisons between the conventional parking system and the proposed parking system. Simulation results prove that the proposed system spends much less time and has higher slot utilization than the conventional system. The simulations also confirm the probability analysis. The future work includes the following:

- relax assumptions (in particular, the fixed arrival rate)

- security and privacy issues

- other performance measures

- extensive simulations

- parking configuration.

Detailed study into these and other areas should be pursed to gain additional insight into parking management. This ultimately can provide intelligent parking services.

\section{REFERENCES}

[1] NYCDCP, "North America CBD Parking Rate Survey Highlights, New York City Department of City Planning," in North America CBD Parking Rate Survey Highlights, August/September 2006.

[2] C. R. Cassady and J. E. Kobza, "A probabilistic approach to evaluate strategies for selecting a parking space," Transportation Science, vol. 32 , no. 1, pp. 30-42, 1998 .

[3] M. Caliskan, D. Graupner, and M. Mauve, "Decentralized discovery of free parking places," in VANET '06: Proceedings of the 3rd international workshop on Vehicular ad hoc networks, New York, NY, USA, 2006, pp. 30-39. 


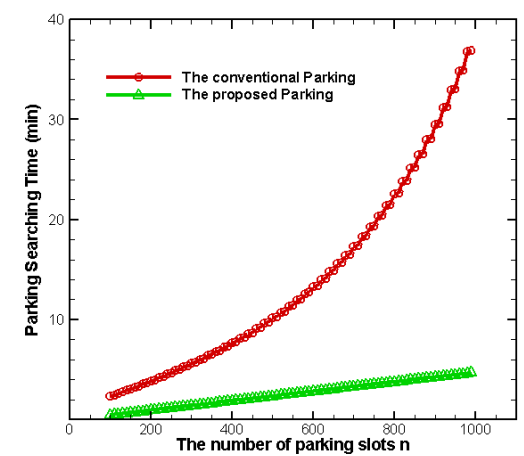

(a) Parking searching time.

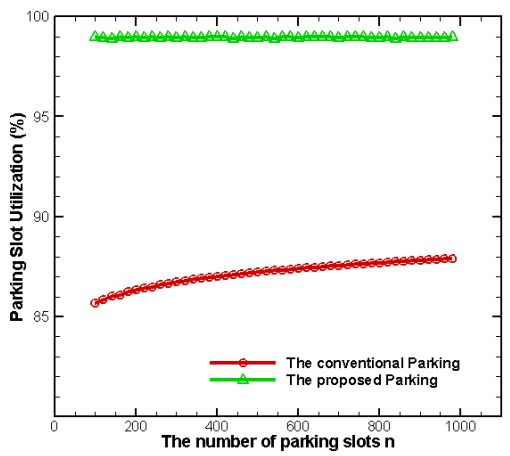

(b) Slot utilization.

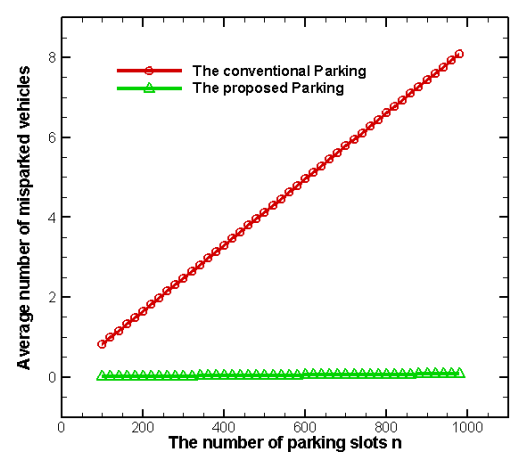

(c) The number of misparked vehicles.

Fig. 5. Comparisons between the conventional and the proposed parking system.

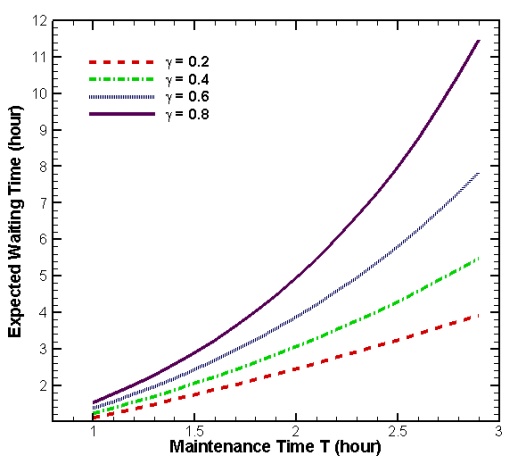

(a) Expected waiting time for maintenance.

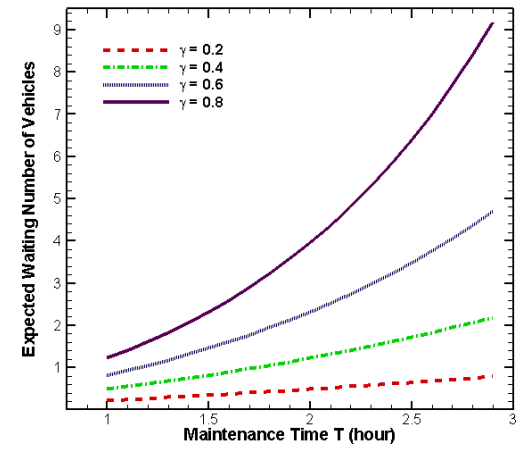

(b) Expected waiting number of vehicles.

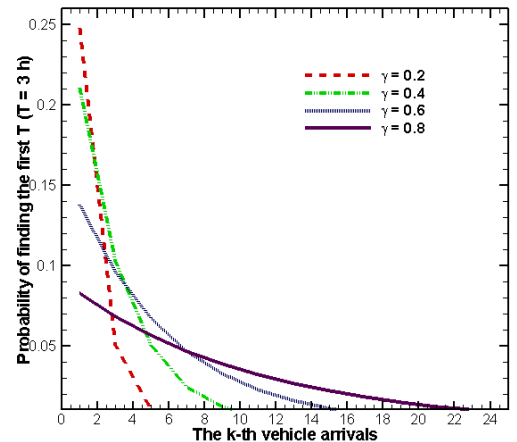

(c) The probability that find the first maintenance time.

Fig. 6. Comparisons of simulation results.

[4] L. E. Y. Mimbela and L. A. Klein, "A summary of vehicle detection and surveillance technologies used in intelligent transportation systems," Nov 2000.

[5] J. Wolff, T. Heuer, H. Gao, M. Weinmann, S. Voit, and U. Hartmann, "Parking monitor system based on magnetic field sensors," in Proceedings IEEE Conf. Intelligent Transportation Systems, Toronto, CA, 2006, pp. 1275-1279.

[6] V. Tang, Y. Zheng, and J. Cao, "An intelligent car park management system based on wireless sensor networks," in Proceedings Int. Sym. Pervasive Computing and Applications, Urumqi, 2006, pp. 65-70.

[7] J. P. Benson, T. O’Donovan, P. O'Sullivan, U. Roedig, and C. Sreenan, "Car-park management using wireless sensor networks," in Proceedings of 31st IEEE Conf. Local Computer Networks, Tampa, 2006, pp. $588-595$.

[8] H. Takizawa, K. Yamada, and T. Ito, "Vehicles detection using sensor fusion," in Proceedings of International Symposium on Intelligent Vehicles (IVS04), Parma, Italy, 2004, pp. 238-243.

[9] Z. F. Zhu, Y. Zhao, and H. Q. Lu, "Sequential architecture for efficient car detection," in Proceedings of IEEE Computer Society Conference on Computer Vision and Pattern Recognition (CVPR 2007), Minneapolis, 2007, pp. 1-8.

[10] S. Funck, N. Mohler, and W. Oertel, "Determining car-park occupancy from single images," in Proceedings of International Symposium on Intelligent Vehicles (IVS04), Parma, Italy, 2004, pp. 325-328.

[11] R. Panayappan, J. M. Trivedi, A. Studer, and A. Perrig, "Vanet-based approach for parking space availability," in VANET '07: Proceedings of the fourth ACM international workshop on Vehicular ad hoc networks, New York, NY, USA, 2007, pp. 75-76.

[12] S. R. Finch, Rényi's Parking Constant. Cambridge, England: Cambridge University Press, 2003.

[13] Y. Asakura and M. Kashiwadani, "Effects of parking availability information on system performance:a simulation model approach," in Proceedings of Vehicle Navigation and Information Systems Conference, Dearborn, MI, Aug 1994, pp. 251-254. 